\title{
Evaluation of Flight Efficiency for Stockholm Arlanda Airport Arrivals
}

\author{
Anastasia Lemetti ${ }^{1}$, Tatiana Polishchuk ${ }^{1}$, Raúl Sáez ${ }^{2}$, and Xavier Prats ${ }^{2}$ \\ ${ }^{1}$ Communications and Transport Systems, ITN , Linköping University, Norrköping, Sweden \\ ${ }^{2}$ Department of Physics, Technical University of Catalonia (UPC), Castelldefels, Barcelona, Spain
}

\begin{abstract}
Analysis of punctuality of airport arrivals, as well as identification of causes of the delays within transition airspace, is an important step in evaluating performance of the Terminal Maneuvering Area (TMA) Air Navigation Services: without knowing the current performance levels, it is difficult to identify which areas could be improved. Deviations from the flight plans is one of the major reasons for arrival delays. In this work, we quantified the impact of the deviations from the flight plans on the fuel burn. One of the main reasons of fuel waste is nonoptimal vertical profiles during the descent phase. We calculated how much extra fuel is wasted due to vertical flight inefficiency within Stockholm TMA.
\end{abstract}

Keywords-Punctuality; Vertical Flight Efficiency; Continuous Descent Operations; Key Performance Indicators

\section{INTRODUCTION}

Air transport punctuality in Europe is one of the major concerns for all the aviation stakeholders. Air traffic delays induce large tactical and strategic costs for airlines, airports and passengers. Delays may also cause environmental damage by increasing fuel consumption and gas emissions [19]. Frequent and long delays generate passenger strong discomfort, which may lead to bad behaviour towards airlines and airport staff, threatening air transportation safety.

Determining causes of aviation delay is essential for formulating and evaluating approaches to reduce air traffic delays. Arrival delays can be a result of trajectories deviations from plans and could lead to extra expenses due to additional fuel burn. Reducing fuel waste has also a significant environmental benefit as it reduces fuel emissions. Another reason of fuel waste is inefficient vertical profiles. At higher altitudes, the air is colder and less dense, which increases aircraft performance. There, aircraft can fly faster and burn less fuel, which represents a double benefit. Thus, the main assumption for the analysis of vertical flight efficiency during descent is that, all other factors being equal, level flight is considered as inefficient.

In this study, we seek to quantify the fuel consumption impact associated with deviations from the flight plans and inefficient vertical flight profiles within Terminal Maneuvering Areas (TMAs). Extra fuel burn, which is directly proportional to the $\mathrm{CO}_{2}$ emissions, is a very good way to estimate flight inefficiencies. It is a more direct estimate on the environmental impact but its computation is more difficult since it requires complex fuel estimation algorithms, since fuel is estimated only from observed surveillance data. In this work, fuel consumption has been computed by using the algorithm proposed in [5] together with the equations from the Base of Aircraft Data (BADA) v4 [12] from EUROCONTROL. More details could be found in section II

The rest of the paper is organized as follows. In Section II we review related work on the topic and provide background information on the methods we use for analysis of the performance of Arlanda airport arrivals. We present the results of data analysis in Section III and summarize our findings in Section IV

\section{BACKGROUND}

This section reviews previous work and provides background information related to evaluation of flight efficiency of airport arrivals.

\section{A. Related Work}

EUROCONTROL developed the methodology used by its Performance Review Unit (PRU) for the analysis of vertical flight efficiency during climb and descent [9]. Performance Review Commission of EUROCONTROL made an assessment of air traffic management in Europe for the year 2018, where among other indicators reviewed air traffic punctuality and vertical flight inefficiency at the top 30 European airports, including Stockholm airport Arlanda [11]. In addition, EUROCONTOL PRU develops and maintains open access cloud based data repositories to enable stakeholders to reproduce the performance review results [23], [24].

Estimation of the flight inefficiencies in terms of extra fuel burn calculated based on the algorithm proposed in [5] was considered in the scope of APACHE project (a SESAR 2020 exploratory research project) [17], [18], but mostly for enroute flight phase. In this work, we apply similar techniques to fuel estimation during the descent phase within TMA.

In [19] fuel consumption is evaluated for terminal areas with a Terminal Inefficiency metric based on the variation in terminal area fuel consumed across flights, reported by a major U.S. airline. Using this metric they quantify the additional fuel burn caused by Air Traffic Management (ATM) delay and terminal inefficiencies.

Furthermore, in [14] and [25], an analysis of fuel savings of Continuous Descent Operations (CDOs) with respect to 
conventional procedures is analyzed. A reduction in fuel consumption of around $25-40 \%$ is achieved by flying CDOs.

Correia [7] analyzed vertical flight efficiency and additional fuel burn in details at Lisbon Airport for the years 2014-2017 and compared statistics with some other European airports, including Arlanda, for the year 2017.

Classification and analysis of causes of airport delays was a topic of interest for many years. In early works [2], [4] weather uncertainties are mentioned as the main contributor to the deviations in airport schedules. Inability of ATM, which limits the airport capacity, to meet the ever raising demand is also one the most significant influencing factor resulting in airport delays [3].

The results of this work create a base for future studies of the impact of different factors such as ATM automation or different weather conditions (wind, convective weather, visibility) on the arrival delays and associated fuel waste.

\section{B. KPIs}

In this work we use the following Key Performance Indicators (KPIs) proposed by the International Civil Aviation Organization (ICAO) [15] to analyze the performance of arrivals in TMA: Arrival punctuality, Level-off during descent.

To assess arrival punctuality we calculate the percent of the flights arriving at the gate on-time (two variants: delayed less than 5 minutes and delayed less than 15 minutes according to the schedule); and to evaluate the vertical flight efficiency we calculate average distance and average time flown in level flight, as summarized in Table I

\section{Vertical Flight Efficiency}

Vertical inefficiencies during the descent phase result from the inability of flights to keep up CDOs. This type of operations enable the execution of a flight profile optimized to the operating capability of the aircraft, giving as a result optimal continuous engine-idle descents (without using speed-breaks) that reduce fuel consumption, gaseous emissions and noise nuisance. If the aircraft levels at intermediate altitudes before landing, this descent is considered as vertical inefficient.

In order to analyze Vertical Flight Efficiency (VFE) we calculate the indicators summarized in Table II. To identify level segments during the descent we use the techniques proposed by EUROCONTROL in [9] with small changes. In order to calculate VFE KPIs inside TMA, we identify the point of the trajectory in which the aircraft enters the TMA and use it as a starting point for the calculations (instead of the Top of Descent, which may lie outside of TMA). A level segment is detected when the aircraft is flying with the vertical speed below the certain threshold. We use the value of 300 feet per minute for this threshold as suggested in [9].

The Continuous Climb Operations(CCO)/CDO Task Force, a group of ATM stakeholders created in 2015 to establish general definitions for measuring $\mathrm{CCO}$ and $\mathrm{CDO}$ operations in Europe, recommends that a single level segment of up to 30 seconds be allowed in the measurement of CDO, and therefore, the time taken for such an event will not be treated as inefficiency. Hence, we consider as level segments only the segments whose time spent in level flight is of minimum 30 seconds. For example, when a level segment of 45 seconds is flown prior to glide slope interception, only 15 seconds of a level segment are counted.

\section{Fuel Burn Calculation}

In this paper, fuel consumption has been computed by using the models provided by BADA v4 [12].

The first expression used, known as the Total-Energy Model, equates the rate of work done by forces acting on the aircraft to the rate of increase in potential and kinetic energy, that is:

$$
(T-D) V_{T A S}=m g \frac{d h}{d t}+m V_{T A S} \frac{d V_{T A S}}{d t}
$$

Here $T$ is the thrust acting parallel to the aircraft velocity vector, $D$ is the aerodynamic drag, $m$ is the aircraft mass, $h$ is the geodetic altitude, $g$ is the gravitational acceleration, $V_{T A S}$ is the true airspeed and $\frac{d}{d t}$ is the time derivative.

The drag force is computed as follows:

$$
D=\frac{1}{2} \cdot \delta \cdot p_{0} \cdot \kappa \cdot S \cdot M^{2} \cdot C_{D}
$$

Here $\delta$ is the pressure ratio, $p_{0}$ is the standard atmospheric pressure at MSL, $\kappa$ is the adiabatic index of air, $S$ is the wing reference area, $M$ is the Mach number and $C_{D}$ is the drag coefficient.

Regarding the drag coefficient $C_{D}$ BADA proposes equations for computing it depending on the aircraft configuration, and modelled as a polynomial of lift coefficient $C_{L}$.

Three separate thrust models are proposed in BADA, depending on the engine type: turbofan, turboprop or piston. Each model includes the contribution from all engines and provides the thrust as a function of airspeed, throttle setting and atmospheric conditions. The general formulation of the thrust force, $T$, is:

$$
T=\delta \cdot W_{m r e f} \cdot C_{T}
$$

Here $\delta$ is the pressure ratio, $m_{r e f}$ is the reference mass (obtained from the Propulsive Forces Model (PFM)), $W_{m r e f}$ is the weight force at $m_{r e f}$ and $C_{T}$ is the thrust coefficient, which is a function of Mach number.

For the three engine types, BADA proposes different equations to compute the thrust coefficient $C_{T}$ depending on the engine rating: maximum climb, maximum cruise, idle and no rating (direct throttle parameter input).

Regarding the fuel consumption, BADA proposes once again a different model depending on the engine type, and also depending on the engine rating. Each model includes the contribution from all engines and provides the fuel consumption as a function of airspeed, throttle parameter and atmospheric conditions. The general formulation of the fuel consumption, $F$, is:

$$
F=\delta \cdot \theta^{\frac{1}{2}} \cdot W_{m r e f} \cdot a_{0} \cdot L_{H V}^{-1} \cdot C_{F}
$$


TABLE I

ARRIVAL PUNCTUALITY AND VFE KPIS

\begin{tabular}{|c|l|}
\hline Notation & Description \\
\hline$K P I 14.1 b$ & percent of arrivals delayed not more than 5 minutes versus schedule \\
$K P I 14.2 b$ & percent of arrivals delayed not more than 15 minutes versus schedule \\
$K P I 19.1$ & average distance flown in level flight inside TMA \\
$K P I 19.2$ & average time flown in level flight inside TMA \\
\hline
\end{tabular}

TABLE II

VFE INDICATORS

\begin{tabular}{|c|c|c|}
\hline Notation & Description & Formula \\
\hline$n_{\text {arrivals }}$ & the total number of arrival flights considered in the analysis & \\
\hline$n_{l}$ & the number of flights considered as level (at least one level segment) & \\
\hline$L_{f}$ & the number of level segments detected in flight $f$ during the descent inside TMA & \\
\hline$D_{f}^{+}$ & the total distance flown by flight $\mathrm{f}$ during the descent inside TMA & \\
\hline$D_{f}$ & the total distance flown level by flight $\mathrm{f}$ during the descent inside TMA & \\
\hline$T_{f}^{+}$ & the total time flown by flight $f$ during the descent inside TMA & \\
\hline$T_{f}$ & the total time flown level by flight $\mathrm{f}$ during the descent inside TMA & \\
\hline$P$ & percent of level flights & $P=\frac{n_{l}}{n_{\text {arrivals }}} * 100$ \\
\hline$L_{a v g}$ & average number of level segments per flight & $L_{a v g}=\frac{\sum_{f} L_{f}}{n_{a r r i v a l s}}$ \\
\hline$D_{f, p e r c}$ & percent of distance flown level by flight $f$ during the descent inside TMA & $D_{f, p e r c}=\frac{D_{f}}{D_{f}^{+}} * 100$ \\
\hline KPI19.1 & average distance flown level per flight inside TMA & $K P I 19.1=\frac{\sum_{f} D_{f}}{n_{\text {arrivals }}}$ \\
\hline$K P I 19.1_{\text {perc }}$ & average percent of distance flown in level flight inside TMA & $K P I 19.1_{\text {perc }}=\frac{\sum_{f} D_{f, \text { perc }}}{n_{\text {arrivals }}}$ \\
\hline$S_{D}$ & distance flown level standard deviation & $S_{D}=\sqrt{\frac{1}{n_{\text {arrivals }}} \sum_{f}\left(D_{f}-D_{\text {avg }}\right)^{2}}$ \\
\hline$T_{f, p e r c}$ & percent of time flown level by flight $f$ during the descent inside TMA & $T_{f, \text { perc }}=\frac{T_{f}}{T_{f}^{+}} * 100$ \\
\hline KPI19.2 & average time flown level per flight inside TMA & $K P I 19.2=\frac{\sum f T_{f}}{n_{\text {arrivals }}}$ \\
\hline KPI19.2 $2_{\text {perc }}$ & average percent of time flown in level flight inside TMA & $K P I 19.2_{\text {perc }}=\frac{\sum_{f} T_{f, p e r c}}{n_{\text {prinols }}}$ \\
\hline$S_{T}$ & time flown level standard deviation & $S_{T}=\sqrt{\frac{1}{n_{\text {arrivals }}} \sum_{f}\left(T_{f}-T_{\text {avg }}\right)^{2}}$ \\
\hline
\end{tabular}

Here $\delta$ is the pressure ratio, $\theta$ is the temperature ratio, $a_{0}$ is the seed of sound at MSL in standard atmosphere, $L_{H V}$ is the fuel lower heating value (obtained from the PFM) and $C_{F}$ is the fuel coefficient, which depends on thrust for non-idle ratings.

For each aircraft model, BADA provides an xml file with the corresponding aircraft performance data. For instance, the coefficients used to compute the thrust coefficient $C_{T}$ of the thrust equation (3) are in this file. With the equations stated above, and the xml files for each aircraft, it is possible to compute the fuel consumption of a trajectory. The process followed is detailed below:

- Thrust computation: if the aircraft is climbing, max climb rating is chosen and the corresponding thrust formula (depending on the engine type) is applied. If the aircraft is descending, an idle rating is assumed. In level-offs, the total-energy model (equation 11) is used in order to compute the corresponding aircraft thrust (drag is computed previously with equation 2 .

- Fuel consumption computation: for non-idle ratings, the thrust computed in the previous step is used to obtain the fuel coefficient $C_{F}$ used in equation 4 . For descents, idle rating is assumed.

It is important to highlight the fact that in this work no wind has been considered when computing the fuel consumption.
Furthermore, a $90 \%$ of the maximum landing mass has been assumed at the destination airport for all aircraft.

1) Generation of $C D O$ trajectories: In order to generate the CDO trajectories an optimal control problem has to be solved. All the details regarding the computation of optimal trajectories can be found in [21].

First, a state vector with the initial conditions is needed. In this paper, it has been chosen as $\boldsymbol{x}=[v, h, s]$, where $v$ is the true airspeed (TAS), $h$ the altitude of the aircraft, and $s$ the distance to go. In order to obtain environmentally friendly trajectories, idle thrust is assumed and speed-brakes use is not allowed throughout the descent. In such conditions, the flight path angle is the only control variable in this problem $(\boldsymbol{u}=[\gamma])$, which is used to manage the energy of the aircraft and achieve different times of arrival at the metering fix with minimum fuel consumption and noise nuisance.

The dynamics of $\boldsymbol{x}$ are expressed by the following set of ordinary differential equations (ODE), considering a point-mass representation of the aircraft reduced to a "gamma-command" model, where vertical equilibrium is assumed (lift balances weight). In addition, the cross and vertical components of the wind are neglected, and the aerodynamic flight path angle is assumed to be small (i.e., $\sin \gamma \simeq \gamma$ and $\cos \gamma \simeq 1$ ): 


$$
\boldsymbol{f}=\left[\begin{array}{c}
\dot{v} \\
\dot{h} \\
\dot{s}
\end{array}\right]=\left[\begin{array}{c}
\frac{T_{i d l e}-D}{m}-g \gamma \\
v \gamma \\
v+w
\end{array}\right]
$$

where $T_{\text {idle }}: \mathbb{R}^{n_{x}} \rightarrow \mathbb{R}$ is the idle thrust; $D: \mathbb{R}^{n_{x} \times n_{u}} \rightarrow \mathbb{R}$ is the aerodynamic drag; $g$ is the gravity acceleration; $w$ is the wind and $m$ the mass, which is assumed to be constant because the fuel consumption during an idle descent is a small fraction of the total $m[6]$. Furthermore, in this work no wind is considered when computing the trajectories.

In this paper, the trajectory is divided in two phases: the latter part of the cruise phase prior the top of descent (TOD), and the idle descent down to the metering fix. Assuming that the original cruise speed will not be modified after the optimization process, the two-phases optimal control problem can be converted into a single-phase optimal control problem as follows:

$$
J=\frac{f}{v_{\text {cruise }}}+\int_{t_{0}}^{t_{f}}\left(f_{\text {idle }}+\mathrm{CI}\right) d t
$$

where $f: \mathbb{R}^{n_{x} \times n_{u}} \rightarrow \mathbb{R}$ and $f_{\text {idle }}: \mathbb{R}^{n_{x}} \rightarrow \mathbb{R}$ are the nominal and idle fuel flow, respectively; and $\mathrm{CI}$ is the cost index, which is a parameter chosen by the airspace user that reflects the relative importance of the cost of time with respect to fuel costs [1]. The CI is estimated by assuming that the aircraft was flying at the optimal speed in the cruise phase, as shown in [20].

To generate the optimum trajectories, five input parameters were used: aircraft model, cruise altitude, distance to go (i.e., the distance remaining to the metering fix by following a given route), speed (i.e., the true airspeed of the aircraft in cruise), and the cost index. Moreover, we needed the aircraft performance model, which was obtained from EUROCONTROL's BADA V4. In the case the aircraft model did not correspond to any of the BADA models, a comparable aircraft in terms of performance and dimensions was used.

\section{RESULTS}

This section presents the results of the flight efficiency evaluation for the Stockholm Arlanda airport arrivals during the year 2018 .

\section{A. Data}

We use two independent databases that provide air traffic data: the Demand Data Repository (DDR2) hosted by EUROCONTROL [13] and the Historical Database of the Opensky Network [16], [22], a crowd-sourced ground sensor network collecting air traffic data from transponder signals that are continuously transmitted by aircraft.

EUROCONTROL offers data in SO6 format that is delimiter separated values files which store flight trajectories (the lists of waypoints containing aircraft position, barometric altitude and identity). DDR2 has two types of these files: SO6 $\mathrm{m} 1$ and SO6 $\mathrm{m} 3$. The first one provides the last submitted flight plans, while the second one consists of the actual trajectories.
We obtain flight plans from the SO6 m1 DDR2 files, but for the historical flight trajectories we use both DDR2 (SO6 m3 file format) and the Historical Database of the Opensky Network.

Opensky provides two types of data: state vectors and tracks. The aircraft state vector is a summary of all tracking information at a certain point in time. This format can provide precise information but is very memory consuming and for that reason not suitable for analysis of the full end-to-end trajectories. Opensky tracks do not contain all information about the flights the database keeps, but rather show the aircraft's general movement pattern as a list of waypoints, similar to EUROCONTROL'S DDR2; however, the choice of waypoints in Opensky and DDR 2 does not match. Opensky data is available to third parties through online databases and APIs.

To estimate the impact of vertical and horizontal flight inefficiencies on the fuel waste we use BADA v4 [12].

\section{B. Data analysis}

In order to evaluate punctuality of arrivals for the major Swedish airport Arlanda, we calculate delay statistics and additional fuel burn due to deviations from the flight plan.

1) Punctuality of arrivals: First, we compared two sets of actual arrival flight times: the one based on DDR2 data and the one provided by Opensky Network, and concluded that due to incompleteness of Opensky network data, DDR2 produces more accurate delay statistics.

Table [II and Figure 1 show the results for the two KPIs calculated using DDR2 data for the year 2018.

TABLE III

ARRIVAL PUNCTUALITY KPIS CALCULATED FOR THE YEAR 2018 BY MONTHS

\begin{tabular}{|c|c|c|}
\hline Months in 2018 & $K P I 14.1 b, \%$ & $K P I 14.2 b, \%$ \\
\hline 01 & 51.4 & 79.5 \\
02 & 50.4 & 77.9 \\
03 & 53.2 & 81.4 \\
04 & 62.9 & 87.8 \\
05 & 66.2 & 88.9 \\
06 & 52.2 & 80.7 \\
07 & 49.7 & 78.6 \\
08 & 57.5 & 83.6 \\
09 & 59.7 & 85.5 \\
10 & 64.4 & 88.9 \\
11 & 63.4 & 88.8 \\
12 & 51.1 & 78.6 \\
\hline
\end{tabular}

2) Vertical Flight Efficiency: Next, we compare vertical flight profiles obtained from DDR m3 data and Opensky data. An example of a vertical flight profile according to the Opensky and DDR m3 data is presented in Figure 2. The Opensky states data is very accurate as it provides altitudes for each second. Calculations based on such a dense data are very memory consuming; still, in the end we used Opensky states to calculate VFE inside TMA, as it only represents a short portion of the total flight.

We use the techniques proposed by EUROCONTROL in [9] with the exception of calculation starting point. We find the 
TABLE IV

VFE INDICATORS AND KPIS CALCULATED FOR THE YEAR 2018 BY MONTHS

\begin{tabular}{|c|c|c|c|c|c|c|c|c|c|}
\hline Months in 2018 & $n_{\text {arrivals }}$ & $P, \%$ & $L_{a v g}$ & $K P I 19.1, \mathrm{NM}$ & $K P I 19.1_{\text {perc }}, \%$ & $S_{D}, \mathrm{NM}$ & $K P I 19.2, \min$ & KPI19.2 $2_{\text {perc }}, \%$ & $S_{T}, \min$ \\
\hline 01 & 7721 & 55 & 1.00 & 2.80 & 5 & 4.62 & 0.72 & 5 & 1.11 \\
\hline 02 & 7419 & 52 & 0.87 & 2.72 & 4 & 4.90 & 0.70 & 5 & 1.18 \\
\hline 03 & 8131 & 56 & 1.08 & 2.99 & 5 & 4.79 & 0.77 & 6 & 1.17 \\
\hline 04 & 8944 & 54 & 1.00 & 2.77 & 4 & 4.97 & 0.69 & 5 & 1.16 \\
\hline 05 & 9552 & 50 & 0.88 & 2.22 & 4 & 4.73 & 0.52 & 4 & 1.01 \\
\hline 06 & 8923 & 51 & 0.90 & 2.68 & 4 & 4.61 & 0.69 & 5 & 1.12 \\
\hline 07 & 8426 & 46 & 0.68 & 2.38 & 4 & 4.21 & 0.61 & 4 & 1.02 \\
\hline 08 & 8915 & 47 & 0.73 & 2.25 & 3 & 3.95 & 0.56 & 4 & 0.94 \\
\hline 09 & 8779 & 48 & 0.79 & 2.28 & 3 & 4.03 & 0.59 & 4 & 1.00 \\
\hline 10 & 9162 & 51 & 0.80 & 2.40 & 4 & 4.10 & 0.64 & 4 & 1.04 \\
\hline 11 & 8558 & 41 & 0.59 & 1.91 & 3 & 3.71 & 0.49 & 3 & 0.91 \\
\hline 12 & 6954 & 49 & 0.73 & 2.68 & 4 & 5.21 & 0.71 & 5 & 1.25 \\
\hline
\end{tabular}

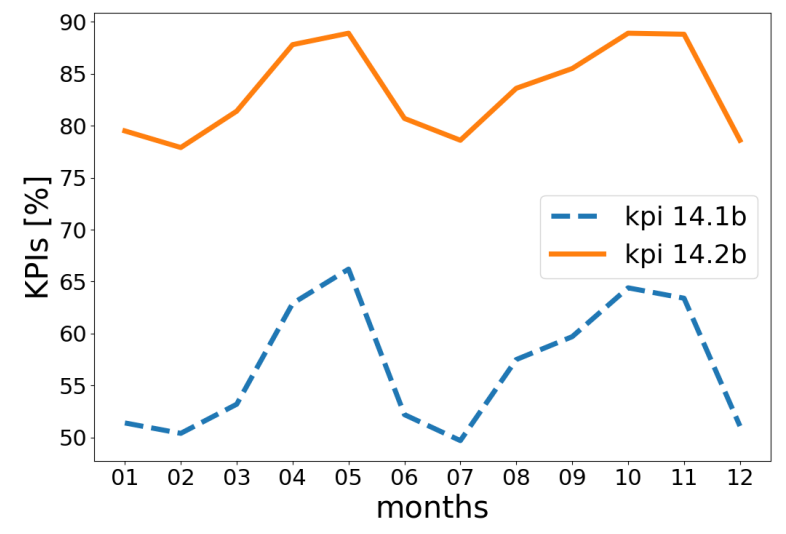

Fig. 1. Percent of the Stockholm Arlanda airport arrivals delayed versus schedule in 2018 , by months.

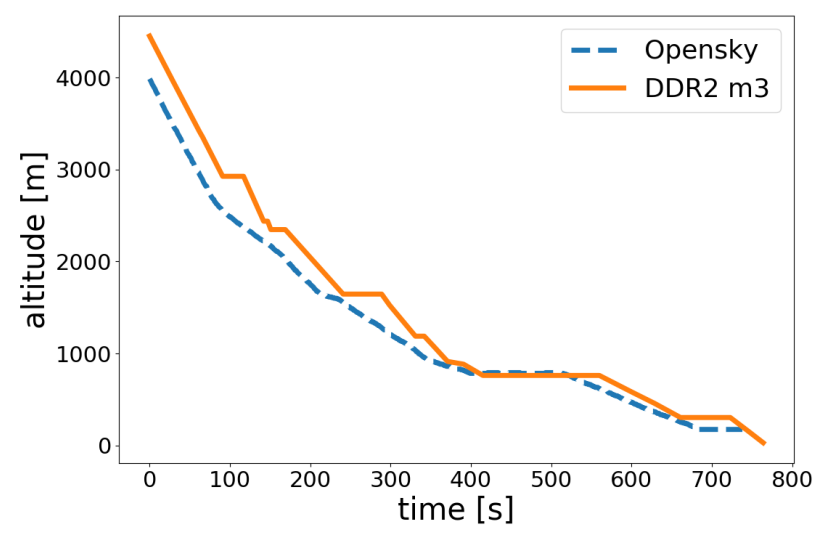

Fig. 2. Vertical profile of the arrival flight with callsign AFL2386 inside TMA from DDR2 m3 and Opensky data on 25.12.2018

point of the trajectory at which the aircraft enters the TMA and use it as a starting point of the trajectory instead of the top of descent. The results are summarized in Table IV] (for the notations, please, refer to Table [I] above), and illustrated in Figure 3 .

We compare the results for the year 2018 against the

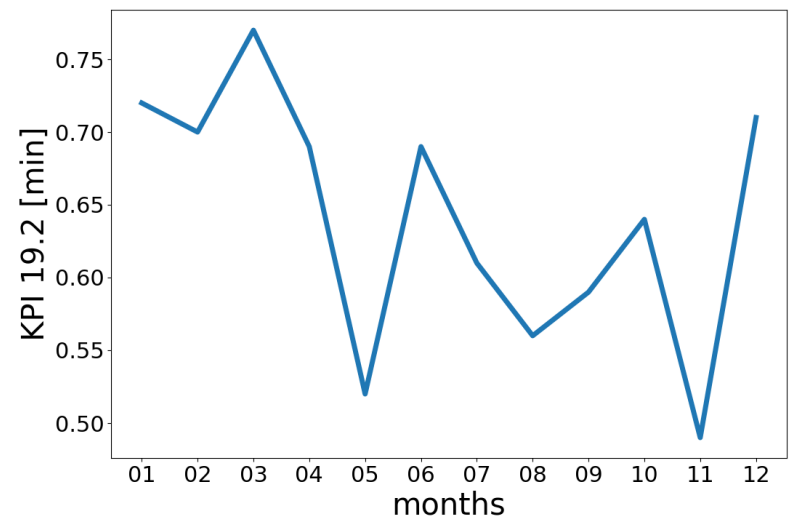

Fig. 3. Average time flown level for arrival flights in Stockholm Arlanda airport in 2018 , by months.

statistics reported in [7] for the year 2017 (Table V), and conclude that the statistics are quite similar for these two consecutive years. Smaller values of VFE KPIs in our calculations most probably do not indicate the improvement of Arlanda arrival flights efficiency, but may result from the different methodology (starting point calculation), different input data (DDR2 vs Opensky states) and slight difference in methods of identifying the level segments.

According to EUROCONTROL's Performance Review Report 2018 [11] average time flown level during descend is 1.1 minute at Arlanda in 2018, which does not contradict our calculations. The report [11] also states that vertical flight efficiency during climb and descent at the top 30 airports remained in 2018 at the same level as in 2017. From that we can conclude that the share of CDO during descend at Arlanda is approximately $50 \%$ ([10] $)$, which coincides to our results.

\section{Additional Fuel Burn}

In order to assess fuel efficiency within Arlanda TMA during the year 2018, we calculate the difference in fuel burn due to deviations from the flight plans (subsection [III-C1), and the fuel waste associated with the vertical flight inefficiency for individual descent profiles within TMA (subsection III-C2). 
TABLE V

COMPARISON OF ARLANDA AIRPORT VFE STATISTICS FOR 2017 (BY [7]) AND 2018 (OUR CALCULATION):

\begin{tabular}{|l|c|c|c|c|c|}
\hline & $n_{\text {arrivals }}$ & $P, \%$ & $L_{\text {avg }}$ & $K P I 19.1, \mathrm{NM}$ & $K P I 19.2 \mathrm{~min}$ \\
\hline $02 / 2017$ & 7332 & 54 & 1.58 & 3.31 & 0.79 \\
$02 / 2018$ & 7419 & 52 & 0.87 & 2.72 & 0.70 \\
$06 / 2017$ & 9317 & 51 & 1.49 & 3.46 & 0.75 \\
$06 / 2018$ & 8923 & 51 & 0.90 & 2.68 & 0.69 \\
\hline
\end{tabular}

Again, for actual trajectories we use both DDR2 and Opensky Network tracks and compare the results for additional fuel burn.

1) End-to-end trajectories: As stated above, the first objective is to compare actual trajectories of the Arlanda arrivals (from origin to destination) with the corresponding flight plans in terms of fuel consumption. In order to do that, two data sources were used. DDR $\mathrm{m} 1$ files correspond to the traffic flight plans, while DDR m3 correspond to the trajectories actually flown. We compare fuel consumption for all the trajectories from the $\mathrm{m} 1$ and $\mathrm{m} 3$ files.

Figure 4 shows the difference in fuel consumption (in percents) between $\mathrm{m} 1$ and $\mathrm{m} 3$ trajectories. Surprisingly, we observed that computations with $\mathrm{m} 1$ data (flight plans) result in a slightly higher fuel consumption than the same calculations with $\mathrm{m} 3$ (actually flown), in most of the cases. This effect can be explained by the shortcuts that ATC tend to give to the flights (as reported in [8]), which decrease the total distance flown and, in the majority of cases, the fuel consumption as well.

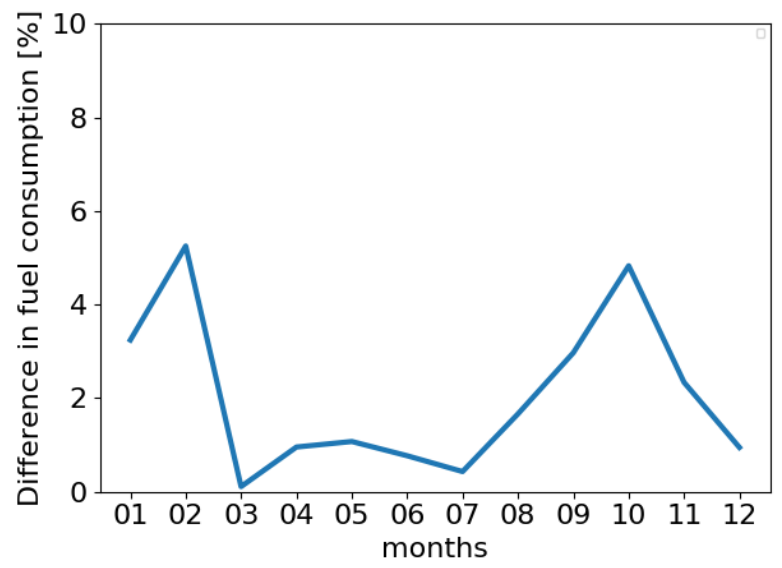

Fig. 4. Difference in fuel consumption for the end-to-end trajectories between the flight plans (DDR m1) and actual flown trajectories (DDR m3), total per month.

2) TMA descents: In TMA, the objective is to compare the fuel consumption of CDO trajectories with the actual flown trajectories (obtained from Opensky tracks and DDR m3 data). The CDO were only optimized for the vertical plane, so the distance to go was obtained from either DDR or Opensky.

Table VI presents the difference in fuel consumption for the same flight (callsign AFL2386, on 25/12/2018), for which the vertical profile was illustrated earlier in Figure 2 , calculated using DDR (m3) and Opensky for actual trajectories and compared to the corresponding CDOs. Opensky data provides more accurate trajectories, where the level segments are calculated with better granularity, which results in higher values of fuel burn.

Another example of the extra fuel consumption due to flying inefficient descent trajectories is shown in Figure 5 where fuel consumption for actual trajectories (DDR2 m3) and CDOs (computed with the trajectory optimization technique described in [20]), are shown for the same callsign AFL2386 during the month of December 2018.

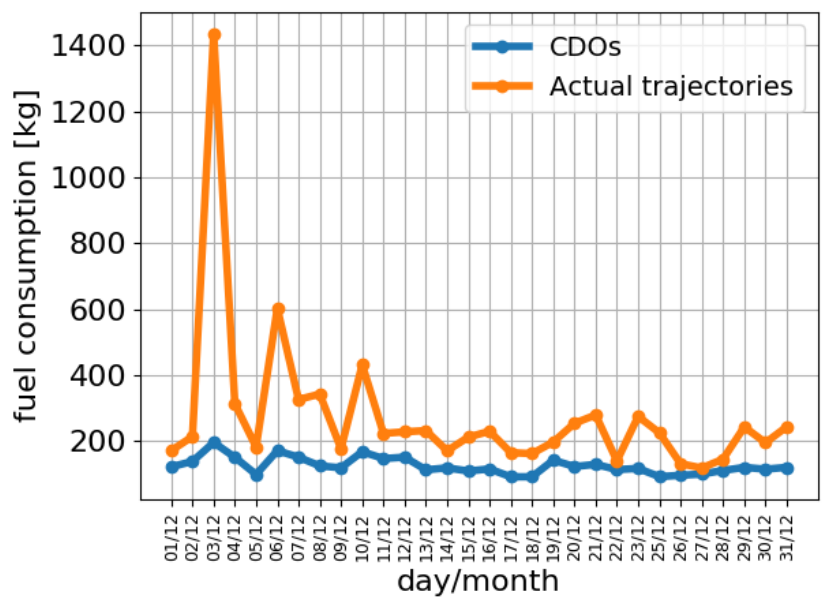

Fig. 5. Fuel consumption for actual flown trajectories (DDR m3) and CDO within TMA for arrival flights in Stockholm Arlanda with callsign AFL2386 during the month of December 2018.

The total values of $8277 \mathrm{~kg}$ and $3773 \mathrm{~kg}$ of fuel are estimated for actual trajectories and CDOs respectively during this month, which demonstrate an improvement of around a $120 \%$ in terms of efficiency when flying CDOs. However, it is not representative enough to analyze only what happens with the same flight over the whole month. In order to understand the result better, we looked at the fuel consumption results per day. As it can be observed in Figure 5, there are two days (more specifically 03/12 and 06/12) where the fuel consumption of the actual trajectories is very high. This is caused by long level segments at low altitudes, which are avoided with CDO. And that is the reason why this high reduction in fuel burn is obtained. During the rest of the days, however, the additional fuel burn remains in the range of $30 \%$ $70 \%$. 
TABLE VI

ADDITIONAL FUEL BURN FOR OPENSKY AND DDR WITH RESPECT TO CDOS FOR AFL2386 ON 25/12/2018

\begin{tabular}{|c|c|c|c|}
\hline DDR & Opensky & CDO (with DDR distance to go) & CDO (with Opensky distance to go) \\
\hline $210 \mathrm{~kg}$ & $245 \mathrm{~kg}$ & $125 \mathrm{~kg}$ & $155 \mathrm{~kg}$ \\
\hline
\end{tabular}

At the end, we calculate the average (over a month) additional fuel burn for all Arlanda airport arrivals during the whole year 2018, first using DDR m3 data for actual trajectories. Figure 6 illustrates the results. We observed that in some months CDO suppose a reduction of fuel consumption of almost a $70 \%$, with an average reduction of approximately $65 \%$ throughout the whole year. It is important to recall that we calculated the fuel inside TMA only; if the whole descent was compared, the difference would have been lower, as leveloffs at lower altitudes are more detrimental for efficiency than those at higher altitudes.

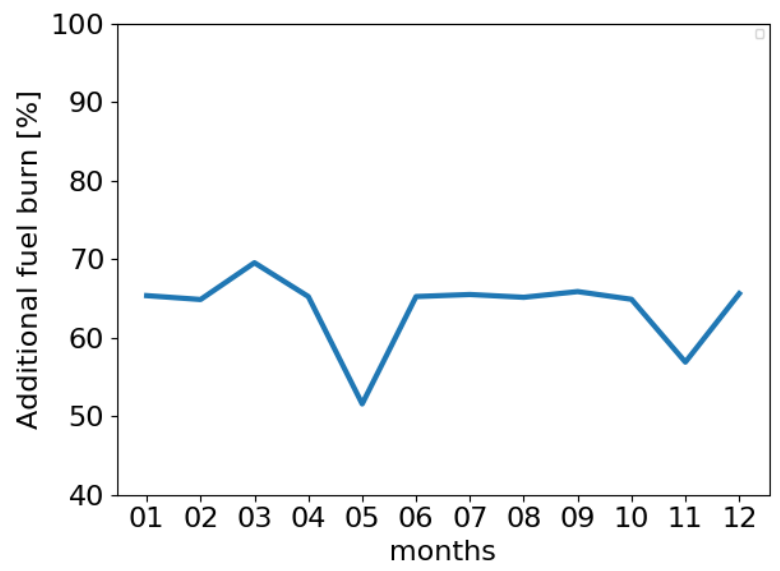

Fig. 6. Additional fuel burn due to inefficient vertical profiles, calculated as the difference between the actual flown and the optimal trajectories, in total fuel consumption per month for the descents inside TMA.

Similar computations have been made by using Opensky data for the month of July. While the fuel consumption is higher than with DDR (about a $6 \%$ more in average), the additional fuel burn with respect to $\mathrm{CDO}$ remains almost the same (around 65\% in average). However, the better data granularity of Opensky data makes it a better option to estimate fuel consumption inside TMA. While DDR usually provides only 3 or 4 segments inside TMA, in Opensky there are about 60-80 waypoints (depending on the trajectory), which makes it more reliable.

\section{CONClusions And Future Work}

In this work, we quantified the impact of the deviations from the flight plans in terms of fuel burn, and also calculated how much extra fuel is wasted due to vertical flight inefficiency within Stockholm TMA.

We investigated Opensky Network data comparing it to EUROCONTROL's DDR2. Although Opensky has certain problems with data integrity and additional methods to detect and filter the different kinds of integrity breaches are needed, it provides the data of high density in form of state vectors with accurate three-dimensional aircraft positions along with precise timestamps for the signal arrivals. A drawback of the Opensky data is the lack of flight identifier, which is critical for flight identification, and aircraft type information, needed for the calculation of fuel consumption. We resolved this problem by merging the data with DDR flight plans (SO6 $\mathrm{m} 1$ files). All in all, we recommend Opensky state vectors as a valuable data source for research with high accuracy demand.

The results of this work create a base for future research; for instance, for the studies of the impact of weather on the flight trajectories (i.e. using the statistics obtained in this work), or for studies focusing on the correlation between the different weather conditions (wind, convective weather, visibility), the arrival delays and associated fuel waste.

\section{REFERENCES}

[1] Airbus. Getting to grips with the cost index - Issue II. Technical Report 2, 1998.

[2] S. Allan, J. Beesley, J. Evans, and S. Gaddy. Analysis of delay causality at newark international airport. In 4th USA/Europe Air Traffic Management R\&D Seminar, pages 1-11. Santa Fe, 2001.

[3] Y. Bai. Analysis of aircraft arrival delay and airport on-time performance. Master's thesis, Tongji University, China, 2004.

[4] R. Beatty, R. Hsu, L. Berry, and J. Rome. Preliminary evaluation of flight delay propagation through an airline schedule. Air Traffic Control Quarterly, 7(4):259-270, 1999.

[5] G. B. Chatterji. Fuel burn estimation using real track data. In 11th AIAA Aviation Technology, Integration, and Operations (ATIO) Conference, including the AIAA Balloon Systems Conference and 19th AIAA LighterThan, page 6881, 2011.

[6] J. P. Clarke, N. T. Ho, L. Ren, J. Brown, K. Elmer, K. F. Zou, C. Hunting, D. McGregor, B. Shivashankara, K. Tong, A. W. Warren, and J. Wat. Continuous descent approach: Design and flight test for Louisville international airport. J. of Aircraft, 41(5):1054-1066, 2004.

[7] P. D. Correia. Analysis of Vertical Flight Efficiency During Climb and Descent. Master's thesis, Técnico Lisboa, 2017.

[8] L. Delgado. European route choice determinants. In 11th USA/Europe Air Traffic Management R\&D Seminar, 2015.

[9] EUROCONTROL. Analysis of vertical flight efficiency during climb and descent.

[10] EUROCONTROL. Performance review report: An assessment of air traffic management in europe during the calendar year 2017.

[11] EUROCONTROL. Performance review report: An assessment of air traffic management in europe during the calendar year 2018 (draft final report for consultation with stakeholders).

[12] EUROCONTROL. User Manual for the Base of Aricraft Data (BADA) Family 4.

[13] EUROCONTROL. DDR2 Reference Manual for General Users 2.9.4, 2017.

[14] H. Fricke, C. Seiss, and R. Herrmann. Fuel and energy benchmark analysis of continuous descent operations. In 11th USA/Europe Air Traffic Management R\&D Seminar, 2015.

[15] KPI Overview. https://www4.icao.int/ganpportal/ASBU/KPI.

[16] OpenSky Network. https://opensky-network.org.

[17] X. Prats, C. Barrado, F. Netjasov, D. Crnogorac, G. Pavlovic, I. Agüi, and A. Vidosavljevic. Enhanced indicators to monitor atm performance in europe. In SIDs 2018, 8th SESAR Innovation Days, 2018.

[18] X. Prats Menéndez, I. Agüi, F. Netjasov, G. Pavlovic, and A. Vidosavljevic. APACHE-Final project results report. 2018. 
[19] M. S. Ryerson, M. Hansen, and J. Bonn. Time to burn: Flight delay, terminal efficiency, and fuel consumption in the national airspace system. Transportation Research Part A: Policy and Practice, 69:286-298, 2014.

[20] R. Sáez, R. Dalmau, and X. Prats. Optimal assignment of 4D close-loop instructions to enable CDOs in dense TMAs. In 37th Digital Avionics Systems Conference, London, England, UK, 2018.

[21] R. Sáez, X. Prats, T. Polishchuk, V. Polishchuk, and C. Schmidt. Automation for Separation with CDOs: Dynamic Aircraft Arrival Routes. In 13th USA/Europe Air Traffic Management R\&D Seminar, 2019.

[22] M. Schäfer, M. Strohmeier, V. Lenders, I. Martinovic, and M. Wilhelm. Bringing Up OpenSky: A Large-scale ADS-B Sensor Network for Research. In IPSN'14, 2014.

[23] E. Spinielli, R. Koelle, K. Barker, and N. Korbey. Open Flight Trajectories for Reproducible ANS Performance Review. In SIDs 2018, 8th SESAR Innovation Days, 2018.

[24] E. Spinielli, R. Koelle, M. Zanin, and S. Belkoura. Initial Implementation of Reference Trajectories for Performance Review. In SIDs 2017, 7th SESAR Innovation Days, 2017.

[25] F. Wubben and J. Busink. Environmental benefits of continuous descent approaches at schiphol airport compared with conventional approach procedures. Technical report, National Aerospace Laboratory (NLR), 2000. 